\title{
SATURATED TWO-PHOTON ABSORPTION BY ATOMS IN A PERTURBER GAS
}

\author{
G. NIENHUIS \\ Fysisch Laboriatorium, Rijksuniversiteit Utrecht, Postbus 80000,3508 TA Utrecht, The Netherlands
}

Received 10 March 1980

\begin{abstract}
We derive a general expression for the two-photon absorption spectrum of a three-state atom excited by two monochromatic radiation fields. Collisional line-broadening effects are incorporated, and the result allows inclusion of profiles with a validity outside the impact limit. Results of previous work are recovered in the appropriate limits. Saturation affects the different lines in the two-photon absorption spectrum in a different fashion.
\end{abstract}

\section{Introduction}

During the past decade the excitation of atoms by absorption of two photons has received a great deal of interest, both as a method of spectroscopy with reduced Doppler broadening [1-3] and as a means of inducing transitions forbidden by one-photon selection rules, in order to study collisional relaxation of populations and coherences of levels that are not easily accessible otherwise [4-6]. In the absence of collisions the two-photon absorption rate attains an appreciable value when the sum frequency of the two photons coincides with the frequency separation between the initial ground state $|g\rangle$ and the final excited state $|e\rangle$, and also when the frequency of one of the two photons is resonant with the onephoton transition from the ground state $|g\rangle$ to the intermediate state $|\mathbf{i}\rangle$. When the absorption lines are broadened by elastic collisions one obtains a third resonance at the transition frequency from $|i\rangle$ to $|e\rangle$ [8]. Moreover, when both photon frequencies are resonant with a one-photon transition the two-photon absorption rate may be enhanced by many orders of magnitude [9]. When Doppler-broadening exceeds collisional broadening, this succession of two resonant one-photon absorptions may give rise to a Dopplerfree two-photon line shape. This may be understood by a simple hole-burning argument. The photons from the first beam excite only the velocity group of atoms whose Doppler-shifted transition frequen- cies coincide with the photon frequency, and the twophoton transition rate is due mainly to this resonant velocity group [7].

In the absence of saturation the two-photon absorption rate is proportional to the product of the intensities of the two light beams. At increasing intensity saturation is easily attained if one of the two frequencies is resonant with the one-photon transition frequency $\omega_{\text {ig }}$ between the intermediate state $|i\rangle$ and the ground state $|g\rangle$. Then the ordinary condition for saturation

$\Omega_{1}^{2} \gtrsim \gamma_{\mathrm{ig}} A_{\mathrm{i}}$

applies, where $\Omega_{1}$ is the Rabi frequency of this resonant transition, $\gamma_{\mathrm{ig}}$ is its homogeneous linewidth and $A_{\mathrm{i}}$ is the rate of spontaneous decay of the state $|\mathrm{i}\rangle$. Saturation of the direct two-photon absorption (when only the sum frequency of the two photons is resonant with $\omega_{\mathrm{eg}}$ ) requires the condition

$\Omega_{1}^{2} \Omega_{2}^{2} \gtrsim \Delta^{2} \gamma_{\mathrm{eg}} A_{\mathrm{e}}$,

where $\Delta$ is the frequency detuning of the photon frequency from the one-photon resonances, $\gamma_{\mathrm{eg}}$ is the homogeneous linewidth of the two-photon transition, $A_{\mathrm{e}}$ is the spontaneous decay rate of $|\mathrm{e}\rangle$ and $\Omega_{1}$ and $\Omega_{2}$ are the two Rabi frequencies. Saturation of the direct process induced by a pulsed laser has been observed in several atoms $[10,11]$. Furthermore a nonresonant high-power light source induces level shifts 
of the order of $\Omega^{2} / \Delta$, which may be observed even in the absence of saturation $[12,13]$.

The theoretical description of two-photon absorption spectra in the steady state has usually focussed on only some of the aspects just mentioned. Making use of two counterpropagating beams with unequal frequencies and low intensities, Bjorkholm and Liao [7] studied the absorption by free atoms (sodium) in the wing of the Doppler-broadened lines. Here the transition region is situated between the velocity selecting two-step process, giving rise to a Dopplerfree two-photon line, and the off-resonance direct process, producing a two-photon absorption line with reduced Doppler broadening. Salomaa and Stenholm [14] described the two-photon absorption profile for two counter-propagating beams with the same frequency that is tuned through the two-photon resonance at $\omega_{\text {eg }} / 2$, where one of the beams has a low intensity. Collisions are accounted for in terms of relaxation constants. Brewer and Hahn [15] have derived a general expression for the steady-state density matrix of a three-state atom excited by two light beams of arbitrary intensity.

In the present paper we give a simple derivation of an expression for the steady-state collision-broadened two-photon absorption spectrum at arbitrary intensities of the two monochromatic light beams. For simplicity we adopt the model of a three-state atom, where each one-photon transition is excited by only one frequency of the two incident radiation beams. The collisional line broadening is expressed in terms of correlation functions which have ordinary normaljzed one-photon line profiles as their Fourier-Laplace transforms. The required approximations are of a similar nature as those adopted before in a theoretical treatment of collision-broadened fluorescence at high intensity [16]. By expressing the high-intensity twophoton absorption spectrum in common one-photon absorption spectra at low intensity, our principal result relates quantities measured in quite different situations, and is therefore liable to experimental verification even when these spectra are hard to calculate. This paper generalizes our previous work on two-photon absorption spectra at low intensities to account for saturation effects. We show that the result describes the different two-photon absorption lines, with their level shifts, their intensity broadening and their saturation broadening in a single unified expression.

\section{Atomic density matrix in the steady state}

A three-state atom in a perturber bath is irradiated by two monochromatic laser beams. The final excited state $|\mathrm{e}\rangle$ and the initial ground state $|\mathrm{g}\rangle$ are both coupled by an electric-dipole transition to the intermediate state $|i\rangle$, by the radiation fields with frequency $\omega_{2}$ and $\omega_{1}$. Each one of the two transitions is excited by only one monochromatic field. This requirement can be satisfied either by choosing the frequencies such that each transition is much nearer to resonance with one of the laser frequencies than with the other, or one may utilize the selection rules and choose properly polarized beams [2]. We assume that the three states are nondegenerate, and we use a classical description of the radiation fields. The interaction $V(t)$ of the atom with the fields in the rotating-wave approximation has the non-vanishing matrix elements

$$
\begin{aligned}
& \langle\mathrm{e}|V(t)| \mathrm{i}\rangle=-\frac{1}{2} \hbar \Omega_{2} \mathrm{e}^{-\mathrm{i} \alpha_{2}-\mathrm{i} \omega_{2} t}=\langle\mathrm{i}|V(t)| \mathrm{e}\rangle^{*}, \\
& \langle\mathrm{i}|V(t)| \mathrm{g}\rangle=-\frac{1}{2} \hbar \Omega_{1} \mathrm{e}^{-\mathrm{i} \alpha_{1}-\mathrm{i} \omega_{1} t}=\langle\mathrm{g}|V(t)| \mathrm{i}\rangle^{*},
\end{aligned}
$$

where $\Omega_{1}=\left|\overrightarrow{\boldsymbol{\mu}}_{\mathrm{ig}} \cdot \overrightarrow{\boldsymbol{E}}_{1} / \hbar\right|$ and $\Omega_{2}=\left|\overrightarrow{\boldsymbol{\mu}}_{\mathrm{ei}} \cdot \overrightarrow{\boldsymbol{E}}_{2} / \hbar\right|$ are the two Rabi frequencies with $\overrightarrow{\boldsymbol{\mu}}$ the atomic electric dipole and $\vec{E}_{1}$ and $\vec{E}_{2}$ the amplitudes of the electric fields. The density matrix $\rho$ of the atom and the perturbers obeys the Liouville equation

$$
\frac{\mathrm{d} \rho(t)}{\mathrm{d} t}=-\mathrm{i} L \rho(t)-\frac{\mathrm{i}}{\hbar}[V(t), \rho(t)] .
$$

The Liouville operator $L$ describes the evolution due to collisions and to spontaneous decay, and is defined

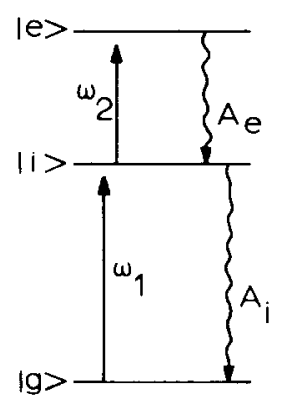

Fig. 1. Level scheme of the model atom with three nondegenerate levels, excited by two monochromatic radiation beams with frequencies $\omega_{1}$ and $\omega_{2}$. Spontaneous decay of the indicated transitions occurs at the rates $A_{\mathrm{e}}$ and $A_{\mathrm{i}}$. 
by

$$
-\mathrm{i} L \rho(t)=-\frac{\dot{1}}{\hbar}[H, \rho(t)]-\Gamma \rho(t),
$$

where $H$ is the quantum-mechanical hamiltonian of the entire particle system, and the operator $\Gamma$ corresponds to spontaneous decay. The state $|\mathrm{e}\rangle$ decays by spontaneous emission to the state $|\mathrm{i}\rangle$ at a rate $A_{\mathrm{e}}$, and the state $|\mathrm{i}\rangle$ decays to $|\mathrm{g}\rangle$ at a rate $A_{\mathrm{i}}$.

The reduced atomic density matrix $\sigma$ of the atom is defined by

$\sigma(t)=\operatorname{Tr}_{\mathrm{p}} \rho(t)$,

where $\operatorname{Tr}_{p}$ denotes the trace over the perturber states. We wish to obtain expressions for the density matrix $\sigma$ in the steady-state limit, which we denote as $\bar{\sigma}$. Its diagonal elements $\bar{\sigma}_{\mathrm{ee}}, \bar{\sigma}_{\mathrm{ii}}$ and $\bar{\sigma}_{\mathrm{gg}}$ are expected to attain constant values. After taking the trace of eq. (2.3) over the states of the perturber bath, we obtain the equations

$$
\begin{aligned}
0= & -A_{\mathrm{e}} \bar{\sigma}_{\mathrm{ee}}+\frac{\mathrm{i}}{2} \Omega_{2} \mathrm{e}^{-\mathrm{i} \alpha_{2}-\mathrm{i} \omega_{2} t} \bar{\sigma}_{\mathrm{ie}}(t) \\
& -\frac{\mathrm{i}}{2} \Omega_{2} \mathrm{e}^{\mathrm{i} \alpha_{2}+\mathrm{i} \omega_{2} t} \bar{\sigma}_{\mathrm{ei}}(t) \\
0= & A_{\mathrm{e}} \bar{\sigma}_{\mathrm{ee}}-A_{\mathrm{i}} \bar{\sigma}_{\mathrm{ii}}+\frac{\mathrm{i}}{2} \Omega_{2} \mathrm{e}^{\mathrm{i} \alpha_{2}+\mathrm{i} \omega_{2} t} \bar{\sigma}_{\mathrm{ei}}(t) \\
& -\frac{\mathrm{i}}{2} \Omega_{2} \mathrm{e}^{-\mathrm{i} \alpha_{2}-\mathrm{i} \omega_{2} t} \bar{\sigma}_{\mathrm{ie}}(t)+\frac{\mathrm{i}}{2} \Omega_{1} \mathrm{e}^{-\mathrm{i} \alpha_{1}-\mathrm{i} \omega_{1} t \bar{\sigma}_{\mathrm{gi}}(t)} \\
& -\frac{\mathrm{i}}{2} \Omega_{1} \mathrm{e}^{\mathrm{i} \alpha_{1}+\mathrm{i} \omega_{1} t \bar{\sigma}_{\mathrm{ig}}(t)} \\
0= & A_{\mathrm{i}} \bar{\sigma}_{\mathrm{ii}}+\frac{\mathrm{i}}{2} \Omega_{1} \mathrm{e}^{\mathrm{i} \alpha_{1}+\mathrm{i} \omega_{1} t \bar{\sigma}_{\mathrm{ig}}(t)} \\
& -\frac{\mathrm{i}}{2} \Omega_{1} \mathrm{e}^{-\mathrm{i} \alpha_{1}-\mathrm{i} \omega_{1} t \bar{\sigma}_{\mathrm{gi}}(t)}
\end{aligned}
$$

where we neglected inelastic collisions. In order to obtain expressions for the offdiagonal elements of $\sigma$ in the steady state, we formally integrate (2.3) to obtain

$\rho(t)=\mathrm{e}^{-\mathrm{i} L \tau} \rho(0)-\frac{\mathrm{i}}{\hbar} \int_{0}^{t} \mathrm{~d} \tau \mathrm{e}^{-\mathrm{i} L \tau}[V(t-\tau), \rho(t-\tau)]$.
This equation yields integral equations for steady-state matrix elements of $\sigma$ alone, provided that we factorize the density matrix $\bar{\rho}$ in the steady state according to

$\bar{\rho}(t)=\bar{\sigma}(t) \rho_{\mathrm{p}}$,

where $\rho_{\mathrm{p}}$ is the density matrix of the perturbers. We introduce correlation functions $C_{\mathrm{ei}}, C_{\mathrm{ig}}$ and $C_{\mathrm{eg}}$ by the definitions

$$
\begin{aligned}
& \operatorname{Tr}_{\mathrm{p}} \mathrm{e}^{-\mathrm{i} L t}\left(|\mathrm{e}\rangle\langle\mathrm{i}| \rho_{\mathrm{p}}\right)=C_{\mathrm{ei}}(t)|\mathrm{e}\rangle\langle\mathrm{i}|, \\
& \operatorname{Tr}_{\mathrm{p}} \mathrm{e}^{-\mathrm{i} L t}\left(|\mathrm{i}\rangle\langle\mathrm{g}| \rho_{\mathrm{p}}\right)=C_{\mathrm{ig}}(t)|\mathrm{i}\rangle\langle\mathrm{g}|, \\
& \operatorname{Tr}_{\mathrm{p}} \mathrm{e}^{-\mathrm{i} L t}\left(|\mathrm{e}\rangle\langle\mathrm{g}| \rho_{\mathrm{p}}\right)=C_{\mathrm{eg}}(t)|\mathrm{e}\rangle\langle\mathrm{g}|
\end{aligned}
$$

These correlation functions decay to zero after a time of the order of the inverse collisional linewidths of the corresponding transitions. We find from (2.8) (2.12) for the offdiagonal elements of $\sigma$ in the steadystate limit:

$$
\begin{gathered}
\bar{\sigma}_{\mathrm{ei}}(t)=\frac{\mathrm{i}}{2} \int_{0}^{\infty} \mathrm{d} \tau C_{\mathrm{ei}}(\tau)\left[\Omega_{2} \mathrm{e}^{-\mathrm{i} \alpha_{2}-\mathrm{i} \omega_{2}(t-\tau)}\left(\bar{\sigma}_{\mathrm{ii}}-\bar{\sigma}_{\mathrm{ee}}\right)\right. \\
\left.-\Omega_{1} \mathrm{e}^{\mathrm{i} \alpha_{1}+\mathrm{i} \omega_{1}(t-\tau)} \bar{\sigma}_{\mathrm{eg}}(t-\tau)\right], \\
\bar{\sigma}_{\mathrm{ig}}(t)=\frac{\mathrm{i}}{2} \int_{0}^{\infty} \mathrm{d} \tau C_{\mathrm{ig}}(\tau)\left[\Omega_{1} \mathrm{e}^{-\mathrm{i} \alpha_{1}-\mathrm{i} \omega_{1}(t-\tau)}\left(\bar{\sigma}_{\mathrm{gg}}-\bar{\sigma}_{\mathrm{ii}}\right)\right. \\
+\Omega_{2} \mathrm{e}^{\left.\mathrm{i} \alpha_{2}+\mathrm{i} \omega_{2}(t-\tau) \bar{\sigma}_{\mathrm{eg}}(t-\tau)\right],} \\
\bar{\sigma}_{\mathrm{eg}}(t)=\frac{\mathrm{i}}{2} \int_{0}^{\infty} \mathrm{d} \tau C_{\mathrm{eg}}(\tau)\left[\Omega_{2} \mathrm{e}^{-\mathrm{i} \alpha_{2}-\mathrm{i} \omega_{2}(t-\tau)} \bar{\sigma}_{\mathrm{ig}}(t-\tau)\right. \\
-\Omega_{1} \mathrm{e}^{\left.-\mathrm{i} \alpha_{1}-\mathrm{i} \omega_{1}(t-\tau) \bar{\sigma}_{\mathrm{ei}}(t-\tau)\right] .}
\end{gathered}
$$

The steady-state matrix elements of $\sigma$ are determined by eqs. (2.5) $-(2.7)$ and (2.11)-(2.13) with the additional normalization condition

$\operatorname{Tr} \bar{\sigma}=\bar{\sigma}_{\mathrm{ee}}+\bar{\sigma}_{\mathrm{ii}}+\bar{\sigma}_{\mathrm{gg}}=1$.

(It should be noticed that the three equations (2.5)(2.7) are not independent since they add up to zero.) We denote the Fourier-Laplace transforms of the 
correlation function as

$F=\int_{0}^{\infty} \mathrm{d} \tau \mathrm{e}^{\mathrm{i} \omega_{2} \tau} C_{\mathrm{ei}}(\tau)=\pi\left[P_{\mathrm{ei}}\left(\omega_{2}\right)-\mathrm{i} Q_{\mathrm{ei}}\left(\omega_{2}\right)\right]$,

$G=\int_{0}^{\infty} \mathrm{d} \tau \mathrm{e}^{\mathrm{i}\left(\omega_{1}+\omega_{2}\right) \tau} C_{\mathrm{eg}}(\tau)$

$$
=\pi\left[P_{\mathrm{eg}}\left(\omega_{1}+\omega_{2}\right)-\mathrm{i} Q_{\mathrm{eg}}\left(\omega_{1}+\omega_{2}\right)\right],
$$

$H=\int_{0}^{\infty} \mathrm{d} \tau \mathrm{e}^{\mathrm{i} \omega_{1} \tau} C_{\mathrm{ig}}(\tau)=\pi\left[P_{\mathrm{ig}}\left(\omega_{1}\right)-\mathrm{i} Q_{\mathrm{ig}}\left(\omega_{1}\right)\right]$.

One directly finds from the general formal expression for absorption or emission spectra [17] that $P_{\text {ig }}\left(\omega_{1}\right)$ is the common normalized absorption profile centered at $\omega_{\text {ig }}$ of the transition from $|g\rangle$ to $|i\rangle$. The function $Q_{\mathrm{ig}}$ is the corresponding dispersion function, that is related to $P_{\mathrm{ig}}$ by the Kramers-Kronig relation

$Q_{\mathrm{ig}}\left(\omega_{1}\right)=\frac{1}{\pi} \mathfrak{P} \int_{-\infty}^{\infty} \mathrm{d} \omega^{\prime} \frac{P_{\mathrm{ig}}\left(\omega^{\prime}\right)}{\omega^{\prime}-\omega_{1}}$.

The other two profile functions and dispersion functions have a similar significance. Furthermore we introduce

$f=\mathrm{e}^{\mathrm{i} \alpha_{2}+\mathrm{i} \omega_{2} t} \bar{\sigma}_{\mathrm{ei}}(t)$,

$g=\mathrm{e}^{\mathrm{i} \alpha_{1}+\mathrm{i} \alpha_{2}+\mathrm{i} \omega_{1} t+\mathrm{i} \omega_{2} t} \bar{\sigma}_{\mathrm{eg}}(t)$,

$h=\mathrm{e}^{\mathrm{i} \alpha_{1}+\mathrm{i} \omega_{1} t} \bar{\sigma}_{\mathrm{ig}}(t)$.

With the substitutions (2.15)-(2.17) and (2.19)(2.21) we obtain the set of coupled linear algebraic equations with constant coefficients

$A_{\mathrm{e}} \bar{\sigma}_{\mathrm{ee}}=\Omega_{2} \operatorname{Im} f$,

$A_{\mathrm{i}} \bar{\sigma}_{\mathrm{ii}}=\Omega_{1} \operatorname{Im} h$,

$f=\frac{\mathrm{i}}{2} F\left[\Omega_{2}\left(\bar{\sigma}_{\mathrm{ii}}-\bar{\sigma}_{\mathrm{ee}}\right)-\Omega_{1} g\right]$,

$g=\frac{\mathrm{i}}{2} G\left[\Omega_{2} h-\Omega_{1} f\right]$,

$h=\frac{\mathrm{i}}{2} H\left[\Omega_{1}\left(\bar{\sigma}_{\mathrm{gg}}-\bar{\sigma}_{\mathrm{ii}}\right)+\Omega_{2} g\right]$.
The solution of this set with the normalization condition (2.14) may be cast in the form

$$
\begin{aligned}
f= & \frac{i}{2} F \Omega_{2}\left[\left(4+G H \Omega_{2}^{2}\right)\left(\bar{\sigma}_{\mathrm{ii}}-\bar{\sigma}_{\mathrm{ee}}\right)\right. \\
& \left.+G H \Omega_{1}^{2}\left(\bar{\sigma}_{\mathrm{gg}}-\bar{\sigma}_{\mathrm{ii}}\right)\right] / K, \\
g= & G \Omega_{1} \Omega_{2}\left[F\left(\bar{\sigma}_{\mathrm{ii}}-\bar{\sigma}_{\mathrm{ee}}\right)-H\left(\sigma_{\mathrm{gg}}-\bar{\sigma}_{\mathrm{ii}}\right)\right] / K, \\
h= & \frac{\mathrm{i}}{2} H \Omega_{1}\left[\left(4+F G \Omega_{1}^{2}\right)\left(\bar{\sigma}_{\mathrm{gg}}-\bar{\sigma}_{\mathrm{ii}}\right)\right. \\
& \left.+F G \Omega_{2}^{2}\left(\bar{\sigma}_{\mathrm{ii}}-\bar{\sigma}_{\mathrm{ee}}\right)\right] / K,
\end{aligned}
$$

$\bar{\sigma}_{\mathrm{ee}}=\left[R^{2}-S T-A_{\mathrm{i}} R\right] / M$,

$\bar{\sigma}_{\mathrm{ii}}=\left[R^{2}-S T-A_{\mathrm{e}} T\right] / M$,

$\bar{\sigma}_{\mathrm{gg}}=\left[R^{2}-S T+A_{\mathrm{e}}(R-T)-A_{\mathrm{i}} S-A_{\mathrm{i}} A_{\mathrm{e}}\right] / M$,

where

$K=4+\Omega_{1}^{2} F G+\Omega_{2}^{2} G H$

and

$$
\begin{aligned}
M= & 3\left(R^{2}-S T\right)+A_{\mathrm{e}}(R-2 T) \\
& -A_{\mathrm{i}}(R+S)-A_{\mathrm{i}} A_{\mathrm{e}},
\end{aligned}
$$

and the functions $R, S$ and $T$ are

$R=\operatorname{Re} \frac{1}{2} \Omega_{1}^{2} \Omega_{2}^{2} F G H / K$,

$S=\operatorname{Re} \frac{1}{2} \Omega_{2}^{2} F\left(4+\Omega_{2}^{2} G H\right) / K$,

$T=\operatorname{Re} \frac{1}{2} \Omega_{1}^{2} H\left(4+\Omega_{1}^{2} F G\right) / K$.

Eqs. (2.27)--(2.37) describe the steady-state density matrix of the atom as a function of the two Rabi frequencies $\Omega_{1}$ and $\Omega_{2}$ and the two radiation frequencies $\omega_{1}$ and $\omega_{2}$ in terms of the profile and dispersion functions.

Two-photon absorption may be monitored in various experimental fashions. The outcome of each type of experiment in the steady state is determined by the density matrix $\bar{\sigma}(t)$. The fluorescent intensity emitted by the excited state is given by

$W_{\mathrm{re}}=A_{\mathrm{e}} \bar{\sigma}_{\mathrm{ee}}$, 
which is the number of emitted photons per time unit per atom. The fluorescent intensity from the intermediate state is

$W_{\mathrm{Fi}}=A_{\mathrm{i}} \bar{\sigma}_{\mathrm{ii}}$.

The number of photons absorbed in a time unit by an atom from the light beam with frequency $\omega_{2}$ is

$W_{\mathrm{A} 2}=\frac{1}{\hbar \omega_{2}} \overline{E_{2}(t) \cdot \frac{\mathrm{d}\langle\mu\rangle}{\mathrm{d} t}}$,

where the brackets denote an average over the steadystate density matrix, and the bar indicates a time average. Only the contribution to the electric dipole from the transition between $|e\rangle$ and $|i\rangle$ contributes to $(2.40)$, and one finds

$W_{\mathrm{A} 2}=\Omega_{2} \operatorname{Im} f$,

which is equal to $W_{\mathrm{Fe}}$ according to (2.22). In the same way one derives for the absorption from the first light beam

$W_{\mathrm{A} 1}=\Omega_{1} \operatorname{Im} h$,

which equals $W_{\mathrm{Fi}}$ according to (2.23). (These statements would no longer be true if the state $|e\rangle$ could decay directly to the ground state $|g\rangle$, e.g. by inelastic collisions.) The most usual way of studying two-photon absorption rates is the observation of the fluorescence emitted by the final excited state $\mid$ e). This rate as a function of $\omega_{1}$ and $\omega_{2}$ is proportional to $\bar{\sigma}_{\mathrm{ee}}\left(\omega_{1}, \omega_{2}\right)$ as given by eq. $(2.30)$, and we shall focus our attention mainly to this expression in several special cases.

The effect of the Doppler shift of moving atoms is easily incorporated if we suppose that the collision cross sections determining the line broadening do not depend on the velocity of the atom and that velocityaltering collisions are sufficiently rare as to allow the absorbing atoms to traverse a distance of the order of a wavelength along a rectilinear path [18]. If the two beams are colinear and have wave numbers $k_{1}$ and $k_{2}$, an atom with a velocity component $v$ parallel to the axis sees the two frequencies $\omega_{1}$ and $\omega_{2}$ as $\omega_{1}-k_{1} v$ and $\omega_{2}-k_{2} v, k$ being taken negative for a beam propagating in the direction opposite to $v$. The Doppler-broadened two-photon absorption spec- trum is then

$\bar{W}_{\mathrm{Fe}}=A_{\mathrm{e}} \int \mathrm{d} v f_{M}(v) \bar{\sigma}_{\mathrm{ee}}\left(\omega_{1}-k_{1} v, \omega_{2}-k_{2} v\right)$,

where $f_{M}(v)=(\beta M / 2 \pi)^{1 / 2} \exp \left(-\beta M v^{2} / 2\right)$ is the Maxwell distribution for atoms with mass $M$.

\section{Saturation broadening and light shifts}

\subsection{Low-intensity limit}

In the low-intensity limit where $\bar{\sigma}_{\text {ee }}$ increases linearly as a function of both intensities, it is sufficient to evaluate (2.30) to second order in both $\Omega_{1}$ and $\Omega_{2}$. By substituting the appropriate orders of $R, S$ and $T$ we find to this order

$\vec{\sigma}_{\mathrm{ee}}=\frac{1}{4 A_{\mathrm{e}}} \Omega_{1}^{2} \Omega_{2}^{2}\left[\frac{1}{2} \operatorname{Re} F G H+\frac{1}{A_{\mathrm{i}}} \operatorname{Re} F \operatorname{Re} H\right]$.

The same result is contained in a previous paper [8] on non-saturated two-photon absorption when the results are specified to the simple model studied in this paper. As shown in ref. 8 , for a frequency $\omega_{1}$ that falls well outside the collisional linewidths, the dispersion function dominates the profiles and we may write

$H\left(\omega_{1}\right) \simeq \mathrm{i} / \Delta_{1}$,

where we introduced for convenience

$\Delta_{1}=\omega_{1}-\omega_{\mathrm{ig}}, \quad \Delta_{2}=\omega_{2}-\omega_{\mathrm{ei}}$.

The analogous far-wing approximations for $F$ and $G$ are

$F\left(\omega_{2}\right) \simeq \mathrm{i} / \Delta_{2}$,

$G\left(\omega_{1}+\omega_{2}\right) \simeq \mathrm{i} /\left(\Delta_{1}+\Delta_{2}\right)$.

When $\omega_{1}$ is well off-resonance, we find for the excited-state population the approximate expression:

$$
\begin{gathered}
\bar{\sigma}_{\mathrm{ee}}=\frac{\pi}{8 A_{\mathrm{e}}} \Omega_{1}^{2} \Omega_{2}^{2}\left[P_{\mathrm{eg}}\left(\omega_{1}+\omega_{2}\right) / \Delta_{1}^{2}\right. \\
\left.+\left(\frac{2 \pi}{A_{\mathrm{i}}} P_{\mathrm{ig}}\left(\omega_{1}\right)-\frac{1}{\Delta_{1}^{2}}\right) P_{\mathrm{ei}}\left(\omega_{2}\right)\right] .
\end{gathered}
$$

This expression as a function of $\omega_{2}$ contains two 
well-separated lines, one at $\omega_{2} \simeq \omega_{\mathrm{eg}}-\omega_{1}$ (or $\Delta_{2} \simeq$ $-\Delta_{1}$ ), corresponding to the direct two-photon absorption process, and one at $\omega_{2} \simeq \omega_{\mathrm{ei}}$ (or $\Delta_{2} \simeq 0$ ), which corresponds to the two-step process with an actual population of the intermediate state $|i\rangle$. When Doppler broadening is accounted for, the first line has a Doppler width of the order of $\left(k_{1}+k_{2}\right) \bar{v}, \bar{v}$ being a typical velocity of the absorbing atom. This Doppler width can be substantially smaller than the common one-photon Doppler broadening when the two beams are counterpropagating, and the two frequencies are about equal. The second line displays the ordinary Doppler width of the order $k_{2} \bar{v}$ [8].

When the ordinary Doppler widths are large compared to the collisional widths, and when the frequency $\omega_{1}$ falls within the Doppler width $k_{1} \bar{v}$ of the line corresponding to the transition from $|g\rangle$ to $\mid i)$, the population of atoms in the intermediate state $|i\rangle$ consists mainly of atoms with the velocity. component

$v=\Delta_{1} / k_{1}$

in the direction of the light beam. Therefore in the two-photon absorption spectrum a Doppler-free line arises at a frequency $\omega_{2}$ which coincides with the Doppler-shifted resonance frequency of this velocity group for the transition from $|i\rangle$ to $|e\rangle$. This line occurs at the frequency

$\omega_{2}=\omega_{\mathrm{ei}}+\Delta_{1} k_{2} / k_{1}$

for the second photon. When the frequency $\omega_{1}$ is tuned outside the Doppler width, this line gets weaker and the two lines at $\omega_{2} \simeq \omega_{\mathrm{ei}}$ and $\omega_{2} \simeq \omega_{\mathrm{eg}}-\omega_{1}$ become visible. For free atoms, when the two-step line at $\omega_{2} \simeq \omega_{\mathrm{ei}}$ is absent [8], the transition from the line at the frequency (3.8) to the direct twophoton line at $\omega_{2} \simeq \omega_{\mathrm{eg}}-\omega_{1}$ has been investigated both theoretically and experimentally by Bjorkholm and Liao [7]. With collisions present, this transition can be evaluated from eq. (3.1), when the profile functions are known.

\subsection{Lowest order in $\Omega_{2}$}

It is illuminating to evaluate the rather intricate expression (2.30) for the steady-state excited-state population in the situation of an intense saturating light beam at frequency $\omega_{1}$, and a second non-satur- ating probe beam at frequency $\omega_{2}$. The lowest order contributing to (2.30) is quadratic in $\Omega_{2}$, and hence linear in the intensity of the second beam. The result may be cast in a form analogous to (3.1) by writing

$\bar{\sigma}_{\mathrm{ee}}=\frac{1}{4 A_{\mathrm{e}}} \Omega_{1}^{2} \Omega_{2}^{2}\left[\frac{1}{2} \operatorname{Re} F^{\prime} G H^{\prime}+\frac{1}{A_{\mathrm{i}}} \operatorname{Re} F^{\prime} \operatorname{Re} H^{\prime}\right]$,

where the modified functions $F^{\prime}$ and $H^{\prime}$ are

$F^{\prime}=F /\left(1+\Omega_{1}^{2} F G / 4\right)$

and

$H^{\prime}=H /\left(1+\Omega_{1}^{2} \operatorname{Re} H / A_{\mathrm{i}}\right)$.

The deviation from the low-intensity limit (3.1) for both radiation beams is contained in the two denominators in (3.10) and (3.11).

The denominator in (3.11) is real, and depends only on $\omega_{1}$. The real part of $H^{\prime}$ gives the saturationbroadened absorption profile for the transition from $|g\rangle$ to $|i\rangle$. This broadening results from the fact that at a given intensity the absorption is more easily saturated close to the resonant value $\omega_{\text {ig }}$ than in the wings of the absorption line.

The denominator in (3.10) is complex, and its effect is most important for values of $\omega_{2}$ near the one-photon resonance $\omega_{\mathrm{ei}}$ (where $F$ is large), or near the two-photon resonance $\omega_{\mathrm{eg}}-\omega_{1}$ (where $G$ is large). If $\omega_{1}$ is near resonance, the effect of this denominator for large values of $\Omega_{1}$ is a splitting of the absorption line for $\omega_{2}$ in two lines that are separated by a distance $\Omega_{1}$. If $\omega_{1}$ is far off resonance, the denominator in (3.10) causes a shift of the two absorption lines for the second photon.

These broadening and shifting effects are best illustrated in the simple case of the impact limit, where the correlation functions $C_{\mathrm{ei}}, C_{\mathrm{eg}}$ and $C_{\mathrm{ig}}$ decay exponentially, and where the functions $F, G$ and $H$ are given by

$F=\left[\gamma_{\mathrm{ei}}-\mathrm{i} \Delta_{2}\right]^{-1}$,

$G=\left[\gamma_{\mathrm{eg}}-\mathrm{i}\left(\Delta_{1}+\Delta_{2}\right)\right]^{-1}$,

$H=\left[\gamma_{\mathrm{ig}}-\mathrm{i} \Delta_{1}\right]^{-1}$,

in which $\gamma_{\mathrm{ei}}, \gamma_{\mathrm{eg}}$ and $\gamma_{\mathrm{ig}}$ are the linewidths, collisional shifts being neglected. The impact limit for collisional 
line broadening at moderate or low perturber densities is valid in a region around the line center of the order of the inverse duration of a collision. After substituting (3.14) we obtain from (3.11)

$\operatorname{Re} H^{\prime}=\gamma_{\mathrm{ig}} /\left[\gamma_{\mathrm{ig}}^{2}+\gamma_{\mathrm{ig}} \Omega_{1}^{2} / A_{\mathrm{i}}+\Delta_{1}^{2}\right]$,

which is a Lorentzian with a saturated linewidth $\gamma_{\text {ig }}^{(s)}$ obeying the equality

$\gamma_{\mathrm{ig}}^{(\mathrm{s})} / \gamma_{\mathrm{ig}}=\left[1+\Omega_{1}^{2} /\left(\gamma_{\mathrm{ig}} A_{\mathrm{i}}\right)\right]^{1 / 2}$.

At a fixed value of $\omega_{1}$ the net effect of this saturation broadening on the absorption spectrum for the probe beam is merely an overall reduction by a factor $\left(1+\Omega_{1}^{2} \operatorname{Re} H / A_{\mathrm{i}}\right)$.

By substitution of (3.12) and (3.13) in (3.10) we obtain

$F^{\prime} G=\left[\left(\gamma_{\mathrm{ei}}-\mathrm{i} \Delta_{2}\right)\left(\gamma_{\mathrm{eg}}-\mathrm{i}\left(\Delta_{1}+\Delta_{2}\right)\right)+\Omega_{1}^{2} / 4\right]^{-1}$.

If $\omega_{1}$ is on resonance, and if $\Omega_{1}$ is larger than $\gamma_{\mathrm{ei}}$ and $\gamma_{\mathrm{eg}}$, this function attains maxima at $\Delta_{2}= \pm \Omega_{1} / 2$. In fig. 2 we show the excited-state population $\bar{\sigma}_{\mathrm{ee}}$ as a function of $\omega_{2}$ for $\Delta_{1}=0$, at various values of the Rabi frequency $\Omega_{1}$ and to second order in $\Omega_{2}$. The occurrence of the line splitting at large values of $\Omega_{1}$ is clearly displayed. This line splitting is a direct manifestation of the dynamic Stark effect [19], and may be easily understood in terms of a dressed-atom picture $[20]$.

Now we consider the case that the detuning $\Delta_{1}$ of the intense beam is large compared to the linewidths and compared to $\Omega_{1}$. We wish to investigate the two absorption lines of the probe field at $\omega_{2} \simeq$ $\omega_{\mathrm{ei}}$ and at $\omega_{2} \simeq \omega_{1}-\omega_{\mathrm{eg}}$. At the first line $\Delta_{2}$ is negligible compared to $\Delta_{1}$, and we may write approximately

$F^{\prime} G \simeq\left[\left(\gamma_{\mathrm{ei}}-\mathrm{i} \Delta_{2}\right)\left(\gamma_{\mathrm{eg}}-\mathrm{i} \Delta_{1}\right)+\Omega_{1}^{2} / 4\right]^{-1}$,

and the position of this line is at the detuning

$\Delta_{2} \simeq \Omega_{1}^{2} / 4 \Delta_{1}$.

From (3.17) we find for the second line the position

$\Delta_{2} \simeq-\Delta_{1}-\Omega_{1}^{2} / 4 \Delta_{1}$.

We see that the intensity of the first light beam enhances the frequency separation of the two absorption lines of the probe beam by a value $\Omega_{1}^{2} / 2 \Delta_{1}$. This
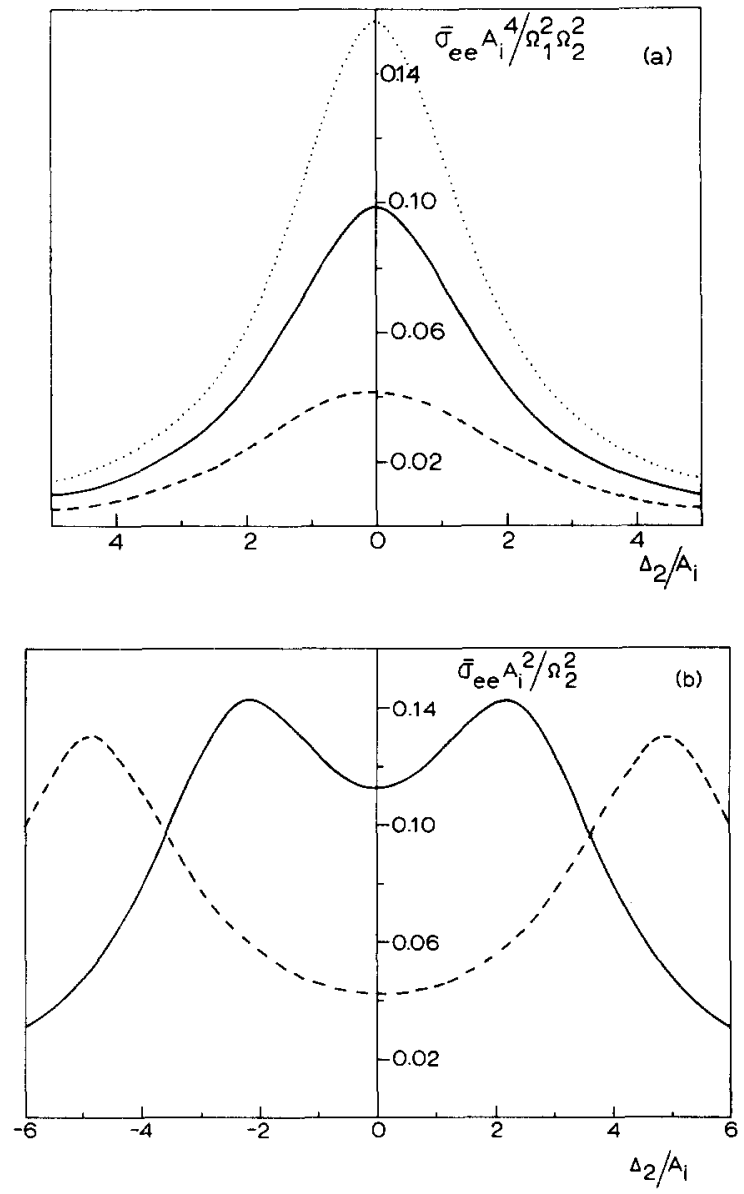

Fig. 2. Stationary excited-state population produced by an intense beam with frequency $\omega_{1}=\omega_{i g}$, as a function of the frequency $\omega_{2}$ of a weak probe beam. The plots are determined by eq. (3.9) where we adopted the impact limit (3.12)-(3.14) for $F, G$ and $H$ with $\gamma_{\mathrm{ei}}=\gamma_{\mathrm{eg}}=\gamma_{\mathrm{ig}}=2 A_{\mathrm{i}}$. Furthermore we assumed $A_{\mathrm{e}}=A_{\mathrm{i}} / 2$. (a) The plotted curves represent $\bar{\sigma}_{\mathrm{ee}} A_{\mathrm{i}}^{4} / \Omega_{1}^{2} \Omega_{2}^{2}$. The dotted curve is the low-intensity limit, which is valid at non-saturating values of $\Omega_{1}$. The solid curve is valid for $\Omega_{1}=A_{i}$, the dashed curve for $\Omega_{1}=2 A_{i}$. (b) The curves represent $\bar{\sigma}_{\mathrm{ee}} A_{\mathrm{i}}^{2} / \Omega_{1}^{2}$. The solid curve holds for $\Omega_{1}=5 A_{i}$, the dashed curve for $\Omega_{1}=10 A_{i}$.

light shift, which is again a manifestation of the dynamic Stark effect, is observable if

$\Omega_{1}^{2} \gtrsim \gamma \Delta_{1}$,

where $\gamma$ is of the order of the collisional widths. For large values of the detuning $\Delta_{1}$ this light shift may be observable even at values of the intensity of the first beam that cause no saturation. 
In fig. 3 we show the steady-state excited-state population $\bar{\sigma}_{\mathrm{ee}}$ as a function of $\omega_{2}$ to lowest order in $\Omega_{2}$, at various values of $\Omega_{1}$ and $\Delta_{1}$. The predicted light shift is clearly visible.

\subsection{First frequency in the far wing}

Another situation of experimental interest that gives rise to a simplification of the general expression (2.30) occurs when the first radiation beam has a frequency $\omega_{1}$ in the far wing of the absorption line centred at $\omega_{\text {ig }}$, so that

$\Delta_{1}>\gamma_{\text {ig }}$.

Furthermore we assume that this detuning is large compared with the two Rabi frequencies

$\Delta_{1} \gg \Omega_{1}, \Omega_{2}$.

We shall demonstrate that in general the two lines in the absorption spectrum as a function of $\omega_{2}$ are shifted from their-low intensity positions $\omega_{\mathrm{ei}}$ and $\omega_{\mathrm{eg}}-\omega_{1}$.

For frequencies $\omega_{2}$ near the one-photon resonance frequency $\omega_{\mathrm{ei}}$ the far-wing limit (3.2) and (3.5) for $H$ and $G$ apply, and we may write

$H \simeq G \simeq \mathrm{i} / \Delta_{1}$

so that $\Omega_{2}^{2} G H$ is small. One finds that the steady-state population of the excited state is given by (2.30), where $R, S$ and $T$ may be approximated by

$R \simeq-\left(\Omega_{1}^{2} \Omega_{2}^{2} / 8 \Delta_{1}^{2}\right) \operatorname{Re} F /\left[1+\mathrm{i} \Omega_{1}^{2} F / 4 \Delta_{1}\right]$,

$S \simeq\left(\Omega_{2}^{2} / 2\right) \operatorname{Re} F /\left[1+\mathrm{i} \Omega_{1}^{2} F / 4 \Delta_{1}\right]$,

$T \simeq\left(\Omega_{1}^{2} / 2\right) \operatorname{Re} H$,

and where $M$ is

$M=3\left(R^{2}-S T\right)-2 A_{\mathrm{e}} T-A_{\mathrm{i}} S-A_{\mathrm{i}} A_{\mathrm{e}}$.

In (3.26) the real part of $H$, which is proportional to the far wing of the profile $P_{\mathrm{ig}}\left(\omega_{1}\right)$, is more important than the imaginary part. One notes that for values of $\omega_{2}$ near to the resonance frequency $\omega_{\mathrm{ei}}, R$ is much smaller than $S$, and $T$ is small compared with $\gamma_{\mathrm{ig}}$. The resulting expression for $\bar{\sigma}_{\text {ee }}$ contains saturation effects due to both intensities. The position of the absorption line is determined by the extreme value of $R$ and $S$ as a function of $\omega_{2}$. One finds that this line near to the



Fig. 3. Same significance as fig. 2 , but at a frequency $\omega_{1}=$ $\omega_{\text {ig }}+10 A_{\mathrm{i}}$ of the intense beam. The dotted curve is the low-intensity limit, the dashed curve holds for $\Omega_{1}=5 A_{\mathrm{i}}$, the solid curve for $\Omega_{1}=10 A_{\mathrm{i}}$.

resonance $\omega_{\mathrm{ei}}$ occurs at the detuning

$\Delta_{2} \simeq \Omega_{1}^{2} / 4 \Delta_{1}$.

Hence the light shift of the absorption line near to the frequency $\omega_{\mathrm{ei}}$ is not affected by the intensity of the second beam. At increasing intensities of the second beam the strength of this absorption line exhibits saturation when $S$ is of the same order of magnitude as $A_{\mathrm{e}}$, so that the intensity-dependent contribution to the denominator (3.27) becomes important. The saturation condition is

$\Omega_{2}^{2} \gtrsim 2 \gamma_{\mathrm{ei}} A_{\mathrm{e}}$,

which can be easily fulfilled even at moderate intensities.

For frequencies $\omega_{2}$ near the two-photon resonance $\omega_{\mathrm{eg}}-\omega_{1}, H$ and $F$ are approximated by their far-wing expression

$H \simeq \mathrm{i} / \Delta_{1} \simeq-F$.

The resulting approximate expressions for $R, S$ and $T$ are

$R \simeq\left(\Omega_{1}^{2} \Omega_{2}^{2} / 8 \Delta_{1}^{2}\right) \operatorname{Re} G /\left[1-\mathrm{i}\left(\Omega_{1}^{2}-\Omega_{2}^{2}\right) G / 4 \Delta_{1}\right]$,

$S \simeq\left(\Omega_{2}^{2} / 2\right) \operatorname{Re} F+R$, 
$T \simeq\left(\Omega_{1}^{2} / 2\right) \operatorname{Re} H+R$.

The approximate result for $\bar{\sigma}_{\text {ee }}$ is found by substituting (3.31)-(3.33) in (2.30), where $M$ is given by (2.34). The order of magnitude of $R, S$ and $T$ strongly depends upon the two Rabi frequencies $\Omega_{1}$ and $\Omega_{2}$, and each one of these quantities can become large compared with $A_{\mathrm{e}}$ or $A_{\mathrm{i}}$ for values of $\Omega_{1}$ and $\Omega_{2}$ obeying the inequalities (3.22). Saturation of the strength of this absorption line occurs as soon as [ one of the intensity-dependent terms in (2.34) gets important. This is the case when any one of the inequalities

$\Omega_{1}^{2} \Omega_{2}^{2} \gtrsim A \gamma_{\mathrm{eg}} \Delta_{1}^{2}$

$\Omega_{2}^{2} \operatorname{Re} F \gtrsim A_{\mathrm{e}}$,

$\Omega_{1}^{2} \operatorname{Re} H \gtrsim A_{\mathbf{i}}$,

is fulfilled. If $\Omega_{1}$ and $\Omega_{2}$ have the same order of magnitude, the onset of saturation will be determined by the condition (3.34). Hence saturation of the direct two-photon process requires higher intensities than are needed for saturation of the two-step process, as expressed by (3.29).

The position of the absorption line corresponding to the direct process may be shifted from its lowintensity position $\omega_{2} \simeq \omega_{\mathrm{eg}}-\omega_{1}$. If we substitute the impact limit (3.13) for $G$ in (3.31), we find that the line centre occurs at the detuning

$\Delta_{2} \simeq-\Delta_{1}+\left(\Omega_{2}^{2}-\Omega_{2}^{2}\right) / 4 \Delta_{1}$.

The light shift is the sum of contribution from each one of the two light beams with opposite signs. By a proper choice of the two intensities this shift can be made to vanish. On the other hand, in general this light shift is observable as soon as exceeds $\gamma_{\mathrm{eg}}$. This is already possible at intensities that are not sufficient to cause saturation of the direct process.

\subsection{Two photons absorbed from a single beam}

So far we discussed the case that the two photons are taken from two different radiation beams. The same theory describes the situation that a single monochromatic laser beam excites the atom by twophoton absorption. Then the general result (2.27)(2.37) for the stationary density matrix still holds, when we substitute

$\omega_{1}=\omega_{2}=\omega$

in the whole of section 2 , and where the ratio $\Omega_{1} / \Omega_{2}$ of the two Rabi frequencies is equal to the ratio of the dipole matrix elements for the two transitions. The various light shifts and broadening effects are well illustrated in this case.

The excited-state population $\bar{\sigma}_{\text {ee }}$ as a function of $\omega$ contains in general three maxima, at $\omega \simeq \omega_{e i}$, $\omega_{\text {eg }} / 2$ and $\omega_{\text {ig. }}$. These three lines are shifted and broadened at high intensities. We assume that these three lines are well-separated, so that

$\left|\omega_{\mathrm{ei}}-\omega_{\mathrm{ig}}\right|>>\gamma$,

where $\gamma$ is of the order of the homogeneous linewidths. Furthermore we assume

$\left|\omega_{\mathrm{ei}}-\omega_{\mathrm{ig}}\right|>>\Omega_{1}, \Omega_{2}$.

For the two lines at $\omega_{\mathrm{ei}}$ and $\omega_{\mathrm{eg}} / 2$ the detuning $\Delta_{1}$ is large, and the theory of the previous subsection applies.

The line at $\omega_{\mathrm{ei}}$ is described by (2.30), where $R, S$, $T$ and $M$ are well approximated by (3.24) $-(3.27)$, with

$\Delta_{1} \simeq \omega_{\mathrm{ei}}-\omega_{\mathrm{ig}}$.

As a result of the light shift (3.28), the position of this line is

$\omega \simeq \omega_{\mathrm{ei}}+\Omega_{1}^{2} /\left[4\left(\omega_{\mathrm{ei}}-\omega_{\mathrm{ig}}\right)\right]$.

The line at $\omega_{\mathrm{eg}} / 2$ is determined by the approximate expressions (3.31)-(3.33) for $R, S$ and $T$, which determine $\bar{\sigma}_{\text {ee }}$ according to (2.30) and (2.34). The detuning $\Delta_{1}$ is

$\Delta_{1} \simeq \omega_{\mathrm{eg}} / 2-\omega_{\mathrm{ig}}=\left(\omega_{\mathrm{ei}}-\omega_{\mathrm{ig}}\right) / 2$.

The light-shifted position of this line is

$\omega \simeq \omega_{\mathrm{eg}} / 2+\left(\Omega_{2}^{2}-\Omega_{1}^{2}\right) /\left[2\left(\omega_{\mathrm{ei}}-\omega_{\mathrm{ig}}\right)\right]$.

For the line at $\omega_{\text {ig }}$ it is the frequency of the second photon that is well off-resonance, with a detuning

$\Delta_{2} \simeq \omega_{\mathrm{ig}}-\omega_{\mathrm{ei}} \cdot$

Then $\bar{\sigma}_{\text {ee }}$ may be approximated in (2.30) the expres- 


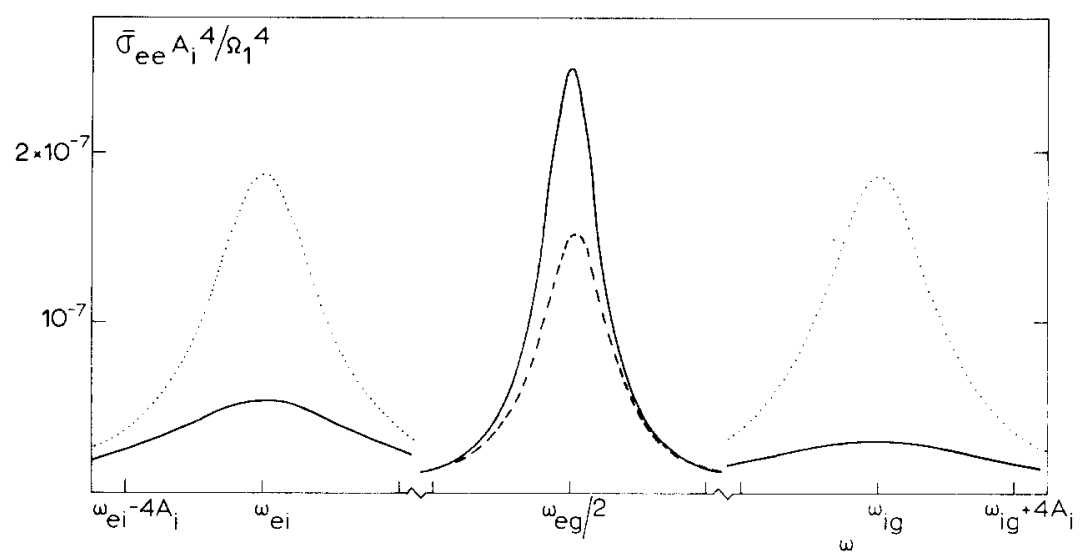

Fig. 4. Stationary excited-state population as a function of the frequency $\omega$ of a single exciting beam. The plotted curves represent $\bar{\sigma}_{\mathrm{ee}} A_{\mathrm{i}}^{4} / \Omega_{1}^{4}$, and are determined by eq. (2.30) with $\omega_{1}=\omega_{2}=\omega, \Omega_{2}^{2}=\Omega_{1}^{2} / 2, A_{\mathrm{e}}=A_{\mathrm{i}} / 2$, and $F, G$ and $H$ are represented by their impact limit (3.12)-(3.14) with $\gamma_{\mathrm{ei}}=\gamma_{\mathrm{eg}}=\gamma_{\mathrm{ig}}=2 A_{\mathrm{i}}$. The value of $\omega_{\mathrm{ig}}-\omega_{\mathrm{ei}}$ is taken to be $1000 A_{\mathrm{i}}$. The dotted curve is the low-intensity limit, the solid curve represents the case $\Omega_{1}^{2}=10 A_{\mathrm{i}}^{2}$. The line centred at $\omega_{\text {eg }} / 2$ for this value of $\Omega_{1}$ is indistinguishable from the low-intensity limit, since it is free of saturation at this intensity. The dashed line represents the situation $\Omega_{1}^{2}=1000 A_{i}^{2}$. At this intensity the lines centred at $\omega_{e i}$ and $\omega_{i g}$ have a maximum value of about $10^{-3}$ times their low-intensity limit, and are not visible on this scale.

sions

$R \simeq-\left(\Omega_{1}^{2} \Omega_{2}^{2} / 8 \Delta_{2}^{2}\right) \operatorname{Re} H /\left[1+\mathrm{i} \Omega_{2}^{2} H / 4 \Delta_{2}\right]$,

$S \simeq\left(\Omega_{2}^{2} / 2\right) \operatorname{Re} F$,

$T \simeq\left(\Omega_{1}^{2} / 2\right) \operatorname{Re} H /\left[1+\mathrm{i} \Omega_{2}^{2} H / 4 \Delta_{2}\right]$,

and the expression (3.27) for $M$. The denominator in (3.45) and (3.47) gives rise to a light shift of this line, and its position is

$\omega \simeq \omega_{\mathrm{ig}}-\Omega_{2}^{2} /\left[4\left(\omega_{\mathrm{ei}}-\omega_{\mathrm{ig}}\right)\right]$.

In fig. 4 we show the behavior of $\bar{\sigma}_{\text {ee }}$ as a function of the frequency $\omega$ of the two absorbed photons, for various intensities of the single exciting beam, in the case that $\Omega_{1}^{2}=2 \Omega_{2}^{2}$, where we adopted the impact limit for the three absorption lines. The curves are based on the general expression (2.30), and display the two-photon excitation spectrum as monitored by the fluorescence emitted by the excited state $|e\rangle$ as a function of the frequency $\omega$. (The number of absorbed photons per time unit is given by

$W_{\mathrm{A}}=A_{\mathrm{i}} \bar{\sigma}_{\mathrm{ii}}+A_{\mathrm{e}} \bar{\sigma}_{\mathrm{ee}}$,

as follows from section 2.) It is obvious from these curves that the lines corresponding to the two-step process are saturated at a lower intensity than the line at $\omega_{\text {ig }} / 2$. The light shifts of the two-step lines are obscured by their saturation broadening.

\section{Conclusion}

We derived a general expression for the elements of the steady-state density matrix of a three-state atom that is irradiated by two monochromatic light beams of arbitrary intensity. The general result as given by eqs. (2.27)-(2.37) contains collisional linebroadening effects that are expressed in terms of common profile functions pertaining to low-intensity experiments. The two-photon absorption rate as monitored by the fluorescence intensity emitted by atoms in the excited state $|e\rangle$ is proportional to $\bar{\sigma}_{\mathrm{ee}}$, as given by (2.30). The Doppler broadening is included by substituting this expression in (2.43). The general result combines and generalizes several previous treatments of two-photon absorption $[7,8$, $14,15]$ and contains saturation broadening and light shifts.

In the low-intensity limit we obtain the simplified expression (3.1), which reproduces our previous result [8]. The result contains the proper twophoton absorption line centred at a sum frequency $\omega_{1}+\omega_{2}$ that coincides with the atomic transition frequency $\omega_{\mathrm{eg}}$. This line corresponds to the direct 
two-photon process, where the intermediate state is only virtually populated. The Doppler width of this line amounts to $\left(k_{1}+k_{2}\right) \bar{v}$, which attains strongly reduced values for counterpropagating beams. Furthermore the spectrum contains absorption lines when one of the two absorbed frequencies coincides with the one-photon transition frequency $\omega_{\mathrm{ei}}$ or $\omega_{\mathrm{ig}}$. These lines correspond to a stepwise excitation process with a real population of the intermediate state, and their Doppler broadening cannot be reduced by a suitable choice of the propagation direction. When both frequencies are near to the atomic transition frequency, the two processes cannot be distinguished.

The saturation effects are quite different for the different lines. When the second radiation beam has a low intensity, the general expression (2.30) simplifies, and the result is written in the form (3.9) that is similar to the low-intensity result (3.1). The denominators in (3.10) and (3.11) contain the effects due to the high intensity of the first beam. In fig. 2 we display a numerical evaluation of eq. (3.9) as a function of $\omega_{2}$ in the case that $\omega_{1}$ coincides with the transition frequency $\omega_{\mathrm{ig}}$, and the quantities $F, G$ and $H$ are represented by the impact limit (3.12)-(3.14) for $\gamma_{\mathrm{ei}}=\gamma_{\mathrm{eg}}=\gamma_{\mathrm{ig}}=2 A_{\mathrm{i}}$. In fig. 2a we plot $\bar{\sigma}_{\mathrm{ee}} / \Omega_{1}^{2} \Omega_{2}^{2}$, which would be independent of $\Omega_{1}$ in the absence of saturation effects. It is mainly the denominator in (3.11) that causes the curves for $\Omega_{1}=A_{i}$ and $\Omega_{1}=$ $2 A_{\mathbf{i}}$ to fall well below the low-intensity limit. At even higher values of $\Omega_{1}$ the denominator in (3.10) gives rise to a splitting of the line, as shown in fig. $2 b$.

Fig. 3 shows the behavior of $\bar{\sigma}_{\mathrm{ee}}$ as given by (3.9) as a function of $\omega_{2}$ at a value of the first frequency $\omega_{1}=\omega_{\mathrm{ig}}+10 A_{\mathrm{i}}$. The line corresponding to the direct process at $\omega_{2} \simeq \omega_{\text {eg }}-\omega_{1}$ and the two-step line at $\omega_{2} \simeq \omega_{\mathrm{ei}}$ are reasonably separated. Saturation effects cause a decay of the strengths of the lines in $\bar{\sigma}_{\text {ee }} / \Omega_{1}^{2}$ at increasing values of $\Omega_{1}$. The shifts of the lines as predicted by eq. (3.19) are clearly displayed in fig. 3 . These light shifts are not affected by collisional effects.

If the first frequency is far off-resonance, a simplified expression holds for the absorption spectrum as a function of the frequency of the second photon at arbitrary intensities of both radiation beams. This result is obtained by substituting (3.24)-(3.27) in (2.30) for the line at $\omega_{2} \simeq \omega_{\mathrm{ei}}$, and by substituting
(3.31) - (3.33) for the line at $\omega_{2} \simeq \omega_{\text {eg }}-\omega_{1}$. The first line can be easily saturated at intensities obeying (3.29), and it has a light shift determined by (3.28). The second line, corresponding to the direct process, requires a much higher intensity for saturation, as expressed by (3.34). Its light shift is given by (3.37). The two radiation fields push the levels $|\mathrm{g}\rangle$ and $|e\rangle$ in the same direction when $\omega_{1}+\omega_{2}$ is near to the transition frequency $\omega_{\mathrm{eg}}$. Hence the light shift is the difference of contributions from the two beams.

The situation that both absorbed photons are taken from the same monochromatic beam constitutes a special case of the general formalism, and it is discussed in section 3.4. When the two one-photon resonances $\omega_{\mathrm{ei}}$ and $\omega_{\mathrm{ig}}$ are well-separated, the twophoton absorption spectrum as a function of the single photon frequency $\omega$ contains three lines at about $\omega \simeq \omega_{\mathrm{ei}}, \omega_{\mathrm{eg}} / 2, \omega_{\mathrm{ig}}$. The light shifts of these lines are given in (3.41), (3.43) and (3.48). The twostep line at $\omega_{\text {ei }}$ saturates at an intensity obeying (3.29), and a similar condition is required for saturation of the two-step line at $\omega_{\text {ig }}$. For these intensities only one of the transitions is saturated, and the intensity dependence of the line strength is of the type $I^{2} /(1+a I)$. Hence for the two-step lines the quadratic rise of the line strength as a function of the incident intensity is followed by a linear increase. The saturation condition for the direct two-photon line at $\omega_{\mathrm{eg}} / 2$ is given by (3.34) where $\Delta_{1}$ is equal to $\left(\omega_{\text {ei }}-\omega_{\text {ig }}\right) / 2$. This saturation condition requires much higher intensities. The line strength has an intensity dependence of the type $I^{2} /\left(1+b I^{2}\right)$. Hence in the saturation region the strength of this line levels off at a constant value.

In fig. 4 we plot $\bar{\sigma}_{\text {ee }} / I^{2}$ as a function of the photon frequency $\omega$. At an intensity obeying $\Omega_{1}^{2}=10 A_{i}^{2}$ the direct two-photon line is still in the low-intensity region, whereas the two-step lines are already strongly saturated.

The general result of this paper is not restricted to the impact limit, and we may substitute in $F, G$ and $H$ profiles and dispersion functions determined by any suitable standard theory of line broadening at low intensities. In principle one might even determine $F, G$ and $H$ by absorption measurements at low intensities, so that our results relate quite different experiments. 
The crucial assumption leading to the general result is the factorization assumption (2.9) for the density matrix in the steady state. Although its validity is not easy to assess theoretically, similar assumptions have led to reasonable results in the past. At very high intensities where the collisional cross sections are affected by the radiation field our assumption is not expected to be justified.

The method that we have presented in this paper is sufficiently versatile to incorporate effects due to the magnetic degeneracy of the atomic states.

\section{References}

[1] L.S. Vasilenko, V.P. Chebotayev and A.V. Shishayev, Sov. Phys. JETP Lett. 12 (1970) 113.

[2] B. Cagnac, G. Grynberg and F. Biraben, J. de Phys. 34 (1973) 845 .

[3] N. Bloembergen and M.D. Levenson, High-Resolution Laser Spectroscopy, K. Shimoda, ed. (Springer, Berlin, 1976) 315 .

[4] F. Biraben, B. Cagnac and G. Grynberg, J. de Phys. 36 (1975) L41.

[5] F. Biraben, B. Cagnac, E. Giacobino and G. Grynberg, J. Phys. B: Atom. Mol. Phys. 10 (1977) 2369.
[6] C.A. van Dijk, P.J.Th. Zeegers, G. Nienhuis and C.Th.J. Alkemade, J. Quant. Spectrosc. Radiative Transfer 20 (1978) 55.

[7] J.E. Bjorkholm and P.F. Liao, Phys. Rev. A14 (1976) 751.

[8] G. Nienhuis and F. Schuller, Physica 94C (1978) 394.

[9] J.E. Bjorkholm and P.F. Liao, Phys. Rev. Lett. 33 (1974) 128.

[10] C.C. Wang and L.I. Davis, Jr., Phys. Rev. Lett. 35 (1975) 650.

[11] J.F. Ward and A.V. Smith, Phys. Rev. Lett. 35 (1975) 653.

[12] P.F. Liao and J.E. Bjorkholm, Phys. Rev. Lett. 34 (1975) 1.

[13] R.G. Brewer and E.L. Hahn, Phys. Rev. A9 (1974) 1479.

[14] R. Salomaa and S. Stenholm, J. Phys. B: Atom. Mol. Phys. 11 (1975) 1795.

[15] R.G. Brewer and E.L. Hahn, Phys. Rev. A1 1 (1975) 1641.

[16] G. Nienhuis and F. Schuller, J. Phys. B: Atom Mol. Phys. (1980) in press.

[17] G. Nienhuis, Physica 66 (1973) 245.

[18] G. Nienhuis, J. Quant. Spectrosc. Radiative Transfer 20 (1978) 275.

[19] S.H. Autler and C.H. Townes, Phys. Rev. 100 (1955) 703.

[20] C. Cohen-Tannoudji, Frontiers in Laser Spectroscopy: Proc. 27th Les Houches Summer School, R. Balian, S. Haroche and S. Liberman, eds. (North-Holland, Amsterdam, 1977) p. 3. 